\title{
Analysis of echocardiographic parameters of cardiac function in patients with acute stroke
}

\author{
Anetta Lasek-Bal ${ }^{1}$, Amadeusz Żak ${ }^{1}$, Piotr Pysz ${ }^{2}$, Przemysław Puz ${ }^{1}$ Zbigniew Gąsior ${ }^{1}$ \\ ${ }^{1}$ Department of Neurology, School of Health Sciences, Medical University of Silesia in Katowice, Poland \\ ${ }^{2}$ Department of Cardiology, School of Health Sciences, Medical University of Silesia in Katowice, Poland
}

\begin{abstract}
Introduction. Cardiologic diagnostics in stroke patients is designed to identify heart disease as a potential cause of stroke. The aim of this study was to evaluate the effect of low ejection fraction (EF) and left ventricular systolic/diastolic dysfunction (LVSD, LVDD) on the neurological state on the 1st day of stroke, as well as post-stroke functional status at 30 days after stroke.

Patients and methods. For a prospective study, 162 stroke patients (mean age 74 years) were qualified. They were analysed according to neurological state on the 1st day of stroke, the results of transthoracic echocardiography, and functional status at 30 days after stroke.

Results. The neurological state on the 1st day after stroke was significantly worse in patients with LVSD. In patients with reduced $\mathrm{EF}$, functional status was significantly worse at 30 days after stroke. Patients with E/A 0.8-2 had a significantly worse functional status compared to patients with $\mathrm{E} / \mathrm{A}<0.8$. Individuals with $\mathrm{E} / \mathrm{A} 0.8-2$ and segmental LVSD or $\mathrm{EF}<50 \%$ had significantly worse functional status compared to patients without LVSD. An independent factor for moderate/severe status was identified: E/A > 0.8 (RR 3.28 [95\% Cl 1.15-9.37]); independent factors for poor functional status were lower EF (RR 4.68 [95\% Cl 1.22-18.00]) and age (RR 4.68 [95\% Cl 1.22-11.00]).

Conclusions. One quarter of patients in the acute phase of stroke have LVSD and/or LVDD. LVSD adversely affects both neurological status in acute stroke as well as functional status in the short-term follow-up. Age at first-in-life stroke incidence and lower $\mathrm{EF}$ are predictors of poor functional status one month after a stroke.
\end{abstract}

Key words: stroke, cardiac dysfunction, fraction ejection, mRankin

(Neurol Neurochir Pol 2019; 53 (2): 144-149)

\section{Introduction}

Cardiologic diagnostics in stroke patients is designed to identify the presence of heart disease as a potential cause of acute cerebral ischaemia and to estimate the risk of stroke recurrence, which is high in patients with myocardial contractile dysfunction and/or thrombosis in the cavities of the heart. Although left ventricular dysfunction (LVD) is considered to be an independent risk factor for stroke, the results of several studies have not shown any significant increase in embolic incidence in patients with LVD [1-3]. A possible explanation for the discrepancies in research results is the type of anticoagulant therapy used that modifies the risk of cerebral and/or systemic embolism to varying degrees. The adverse effect of LVD on the status of patients with chronic stroke has been reported. However, the importance of LVD for neurological status in the early period of stroke onset has not been clearly established. Given the haemodynamic effects of LVD in the cerebral circulation, the effect of worsening hypoperfusion in the ischaemic zone is to be expected.

The aim of this study was to evaluate the potential effect of low ejection fraction (EF), and segmental systolic dysfunction and left ventricular diastolic dysfunction, on the neurological state of patients on the 1st day after stroke onset, as well

Address for correspondence: Anetta Lasek-Bal, Department of Neurology, School of Health Sciences, Medical University of Silesia in Katowice, Poland, e-mail: abal@sum.edu.pl 
as post-stroke functional status at 30 days after stroke. An additional objective was to evaluate the significance of other cardiac echocardiographic parameters in acute stroke and the short-term prognosis in this group of patients.

\section{Methods}

For a prospective study covering the period 2015-2016 in the Neurology Department, 162 patients ( 77 women, $85 \mathrm{men}$ ), mean age 74 years $(71.9 \pm 12.6)$ [25-96] were classified, with the first clinically manifested stroke identified according to the WHO clinical criteria and visible in neuroimaging (computed tomography and/or magnetic resonance imaging of the head) acute ischaemic lesion of the brain [1]. Other main inclusion criteria were: time from onset of stroke symptoms to hospital admission $\leq 24$ hours; and the pre-stroke status according to the modified Rankin Scale $(\mathrm{mRS}) \leq 1$ point.

All patients included in the study were analysed according to:

- age when they had their first-in-life stroke

- presence of comorbidities such as atrial fibrillation (AF), arterial hypertension $(\mathrm{AH})$, coronary heart disease (CHD), diabetes mellitus (DM) and lipid disorders (LD), > 70\% atherosclerotic carotid stenosis (ipsilaterally to the ischaemic brain lesion)

- neurological state on the 1st day after stroke onset evaluated according to NIHSS (National Institute of Health Stroke Scale) [3]

- anatomic location of stroke (total anterior cerebral infarct, TACI); partial anterior CI (PACI), lacunar CI (LACI), and posterior CI (POCI)

- early therapeutic management in the acute stage of stroke (thrombolytic intravenous (rtPA iv), endovascular thrombectomy, antiplatelet treatment)

- the results of transthoracic echocardiography (TTE) within the first two days of stroke onset, including heart cavity dimensions, left ventricular contractility and valvular function evaluated according to the recommendations of the European Association of Cardiovascular Imaging (EACVI)

- functional status at 30 days after stroke according to the $\mathrm{mRS}$ scale (based on information obtained from the patient and/or carer during a phone call [4].

Diagnosis of AH was consistent with the recommendations of the European Society of Cardiology (ESC), DM was diagnosed according to the criteria of the Diabetes Association, dyslipidaemia was defined according to the ESC recommendations (Guidelines for the Management of Dyslipidaemias) [4-6].

The degree of stenosis of common carotid artery and/or internal carotid artery was assessed according to the NASCET criteria [7]. The TTE was performed using the Philips Epiq 7.

The patients were divided into groups depending on the presence of segmental contractility abnormalities (patients with present $v s$ absent contractility dysfunction), ejection fraction $(\mathrm{EF}<50 \% v s \geq 50 \%)$, left atrial size (LA $\leq 40 \mathrm{~mm} v s>40 \mathrm{~mm})$, left ventricular end-diastolic dimension (EDD $<56 \mathrm{~mm}$ $v s \geq 56 \mathrm{~mm}$ ), mitral regurgitation degree (MR $1+2 v s 3)$, aortic stenosis degree (AS $1+2 v s 3$ ), aortic regurgitation degree $(\mathrm{AR} 1+2 v s 3)$, relative wall thickness (patients with present $v s$ absent hypertrophy) or eccentric hypertrophy (patients with present $v s$ absent eccentric hypertrophy). In these groups, mean scores for NIHSS (neurological state on the 1st day after stroke onset) and $\mathrm{mRS}$ (functional status at 30 days after stroke onset) were calculated, and a comparison was made between subgroups. Next, 113 patients without atrial fibrillation were divided into three groups depending on the rate of mitral inflow $\mathrm{E}$ to A: group A: E/A ratio $\leq 0.8$ - patients with impaired LV relaxation; group $B$, which included patients with no abnormalities or with pseudo-normal pattern of mitral inflow: E/A 0.8-2; and group C - patients with restricted mitral inflow pattern: $\mathrm{E} / \mathrm{A}>2$. In these groups, mean scores for NIHSS were calculated (on the 1st day after stroke), and mRS (at 30 days after stroke), and a comparison was made between the groups.

For the identification of patients with left ventricular diastolic dysfunction with pseudo-normal pattern of mitral inflow, patients in group B were divided into two subgroups according to the following criteria: $\mathrm{B} 1$ - patients with E/A ratio $0.8-2$, present segmental contractility dysfunction and/ or $\mathrm{EF}<50 \%$; subgroup B2 - patients with E/A 0.8-2, absence of segmental contractility dysfunction and normal EF (> 50\%). Neurological status according to NIHSS (24h after stroke onset), and functional status according to mRS (30 days after stroke onset) were assessed in subgroups B1 and B2 and the mean values obtained were compared. Multi-factorial analysis was performed to determine independent factors of bad neurological status on the 1st day (NIHSS $>10$ points) and poor prognosis defined as the patient receiving 3-6 points on mRankin Scale at 30 days after stroke. The following parameters were included in the analysis: age, sex, arterial hypertension, diabetes mellitus, coronary heart disease, atrial fibrillation, lipid abnormalities, $>70 \%$ carotid stenosis, location of stroke (PACI, TACI, POCI, LACI), therapeutic management in the acute stage of stroke (reperfusion therapy- rtPA or/and thrombectomy, antiplatelet therapy without reperfusion strategies), EF reduction, size of LA, AS, MR, RWT, EDD, and NIHSS $24 \mathrm{~h}$ after stroke for the identification of prognostic factors for one month after the onset of stroke.

All statistical analyses were performed using STATISTICA 8.0 PL software. Chi-square and Student's tests were used for categorical variables. The Mann-Whitney U test was used to compare the study groups and subgroups for the nonparametric distribution of some of the parameters. Finally, an analysis was made using a single and multi-factorial method of nonlinear estimation - logistic regression (STATISTICA 5.0PL) - to identify independent factors for moderate/severe neurological state and post-stroke disability at 30 days after stroke. $\mathrm{P}<0.05$ was considered statistically significant.

Ethical approval was not necessary for preparation of this article. 


\section{Results}

The baseline characteristics of patients enrolled in the study are presented in Table 1.

The neurological state on the 1st day after stroke was significantly worse in patients with left ventricular contractile dysfunction. In patients with reduced ejection fraction assessed within the first two days after stroke functional status at 30 days after stroke was significantly worse. In patients with left ventricular hypertrophy and/or left atrial enlargement, both neurological and functional status was worse, but the differences relative to patients with normal $\mathrm{LV}$ and $\mathrm{LA}$ were not statistically significant (Tab. 2).

Patients with E/A ratio $0.8-2$ had a significantly worse functional status after one month of stroke onset compared to patients with $\mathrm{E} / \mathrm{A}<0.8$ (Tab. 3).

Patients with E/A 0.8-2 and LV contractile dysfunction LVSD and/or EF $<50 \%$ had significantly worse outcomes in functional status assessment compared to patients without $\mathrm{LV}$ systolic dysfunction (Tab. 4).

In multi-factorial analysis, only one independent factor - E/A > 0.8 (RR 3.28 [95\% CI 1.15-9.37] p < 0.0208) was identified for moderate/severe neurological status (NIHSS $>10$ points) on the 1st day of stroke. There were two independent factors for poor functional status prognosis (3-6 mRankin) at 30 days after stroke: lower EF (RR 4.68 [95\% CI 1.22-18.00], p 0.0186) and age RR 4.68 [95\% CI 1.22-11.00], p < 0.0179. The other analysed clinical and echocardiographic parameters were not independent factors for patients's state.

\section{Discussion}

In the present study, abnormal echocardiographic parameters indicating structural and/or functional myocardial dysfunction were found in $23 \%$ of patients. The most important result of the present study is the finding of unfavourable effects of both systolic and diastolic left ventricular dysfunction on the course of stroke. The significance of selected LVD parameters for stroke patient status varies and is marked both on the 1st day after stroke as well as one month after the onset of symptoms. Knowledge of the presence of cardiac dysfunction in a stroke patient is not only important for prognosis but may be crucial for planning early and subsequent rehabilitation.

Population studies estimate the incidence of left ventricular dysfunction to be about $15-25 \%$, including $0.9-6 \%$ of patients who are asymptomatic [8-11]. LVD is more common in people $>65$ years of age, and in the subpopulation of patients with arterial hypertension and diabetes [8]. Older age, hypertension and diabetes also increase the risk of stroke. LVD is a consequence of cardiomyocyte death because of oxidative stress originating in the cardiomyocytes as a result of ischaemia, infection, or toxicity [12]. Clinical manifestations of heart failure such as dyspnoea and reduced exercise tolerance are more common in left ventricle diastolic dysfunction (LVDD).
Table 1. Characteristics of the patients

\begin{tabular}{lc}
\multicolumn{1}{c}{ Parameter } & Group $\mathbf{n}=\mathbf{1 6 2}$ \\
\hline Age & $71.9 \pm 12.6$ \\
Sex F/M & [25-96] median 74 \\
DM & $77 / 85$ \\
AH & $46(28.4 \%)$ \\
AF & $143(88.3 \%)$ \\
AF de novo & $49(30.2 \%)$ \\
MI & $14(8.6 \%)$ \\
Location of stroke & $28(17.3 \%)$ \\
TACl & \\
PACl & $69(42.6 \%)$ \\
LACl & $40(24.7 \%)$ \\
POCl & $44(27.2 \%)$ \\
rtPA-therapy & $9(5.6 \%)$ \\
thrombectomy & $38(23.6 \%)$ \\
antiplatelet without reperfu- & $6(3.7 \%)$ \\
sion therapy & $117(72.7 \%)$ \\
\hline
\end{tabular}

$\mathrm{F}$ - female, $\mathrm{M}$ - male, $\mathrm{DM}$ - diabetes mellitus, $\mathrm{AH}$ - arterial hypertension, $\mathrm{AF}$ - atrial fibrillation, $\mathrm{MI}$ - past myocardial infarct, $\mathrm{PACl}$ - partial anterior cerebral infarct, $\mathrm{TACl}$ - total anterior cerebral infarct, $\mathrm{POCl}$ - posterior cerebral infarct, $\mathrm{LACl}$ - lacunar cerebral infarct, rtPA — recombined tissue plasminogen activator

This means that many patients may be unaware of their cardiac contractility dysfunctions, and according to the Framingham study the mortality rate of patients with left ventricular systolic dysfunction is higher, and it constitutes $17.9 \%$ (compared to $8.9 \%$ in patients with diastolic dysfunction, and $3.7 \%$ in the control group) [13].

Based on a multivariate analysis, the presence of moderate or severe LVSD in two-dimensional echocardiography has been shown to increase the prevalence of an acute embolic event, including stroke [14, 15]. Contractile disorders have also been associated with the risk of stroke recurrence [16]. Park et al. [17] reported LVD in more than half of stroke patients, including profound dysfunction in every tenth stroke patient. In such cases, LVD may impede functional recovery of stroke survivors due to their lower exercise capacity.

An adverse implication of limited rehabilitation, regardless of other comorbid diseases, may be the worse post-stroke functional state of stroke survivors. The results of this study indicate that the neurological status on the 1st day after stroke in patients with LVSD was significantly worse than in patients with normal $\mathrm{LV}$ function. The consequence of haemodynamic insufficiency associated with LVSD is cerebral hypoperfusion. This impedes the already impaired blood flow in the penumbra area surrounding the cerebral infarction lesion. Other coexisting disorders such as impaired cerebral autoregulation, endothelial dysfunction, prothrombotic state, and infection additionally adversely affect the evolution of the cerebral ischaemic lesion [18-20]. Also, the effect of reperfusion in 
Table 2. Neurological status on the 1st day and functional status at 30 days after stroke onset according to selected echocardiographic parameters

\begin{tabular}{|c|c|c|c|c|}
\hline Parameter, n & NIHSS & $\mathbf{p}$ & mRankin & $\mathbf{p}$ \\
\hline \multicolumn{5}{|l|}{ Segmental contractility LV } \\
\hline normal, $\mathrm{n}=127$ & $3.74 \pm 4.65$ & 0.013 & $2.29 \pm 1.76$ & 0.10090 \\
\hline abnormal, $n=35$ & $5.40 \pm 4.60$ & & $2.83 \pm 1.82$ & \\
\hline \multicolumn{5}{|l|}{ EF } \\
\hline$<50, n=23$ & $5.67 \pm 5.19$ & 0.054 & $3.08 \pm 1.67$ & 0.0386 \\
\hline$\geq 50, n=139$ & $3.93 \pm 4.69$ & & $2.31 \pm 1.79$ & \\
\hline \multicolumn{5}{|l|}{ EDD } \\
\hline$<56, n=142$ & $4.22 \pm 4.82$ & 0.379 & $2.40 \pm 1.79$ & 0.4508 \\
\hline$\geq 56, n=20$ & $5.27 \pm 4.88$ & & $2.82 \pm 1.83$ & \\
\hline \multicolumn{5}{|l|}{ LA } \\
\hline$<40, n=98$ & $4.20 \pm 4.96$ & 0.592 & $2.30 \pm 1.78$ & 0.4152 \\
\hline$\geq 40, n=64$ & $4.31 \pm 4.71$ & & $2.53 \pm 1.85$ & \\
\hline \multicolumn{5}{|l|}{ MR } \\
\hline $1-2, n=104$ & $4.22 \pm 4.84$ & 0.811 & $2.29 \pm 1.75$ & 0.6380 \\
\hline $3, n=13$ & $7.00 \pm 9.64$ & & $2.67 \pm 2.08$ & \\
\hline \multicolumn{5}{|l|}{ AS } \\
\hline $1-2, n=19$ & $5.56 \pm 6.26$ & 0.963 & $2.38 \pm 2.00$ & 0.4372 \\
\hline $3, n=9$ & $3.00 \pm 1.63$ & & $3.50 \pm 0.58$ & \\
\hline \multicolumn{5}{|l|}{ AR } \\
\hline $1-2, n=63$ & $4.60 \pm 5.47$ & - & $2.56 \pm 1.80$ & - \\
\hline $3, n=0$ & - & & - & \\
\hline \multicolumn{5}{|c|}{ Concentric RWT hypertrophy } \\
\hline absent, $n=101$ & $4.46 \pm 4.92$ & 0.4393 & $2.32 \pm 1.75$ & 0.3183 \\
\hline present, $\mathrm{n}=61$ & $4.02 \pm 4.68$ & & $2.62 \pm 1.86$ & \\
\hline \multicolumn{5}{|c|}{ Eccentric RWT hypertrophy } \\
\hline absent, $n=108$ & $4.31 \pm 5.13$ & 0.3120 & $2.48 \pm 1.79$ & 0.5536 \\
\hline present, $n=54$ & $4.27 \pm 4.01$ & & $2.31 \pm 1.81$ & \\
\hline
\end{tabular}

NIHSS - National Institute of Health Stroke Scale, LV — left ventricle, EF — ejection fraction, EDD — end-diastolic dimension, LA — left atrium, MR — mitral regurgitation, AS — aortic stenosis, AR — aortic regurgitation, RWT — relative wall thickness

Table 3. Neurological status on the 1st day and functional status at 30 days after stroke onset depending on the velocity of mitral inflow $\mathrm{E} / \mathrm{A}$

\begin{tabular}{|c|c|c|c|c|c|c|}
\hline & $\begin{array}{c}\text { Group } 1 \\
E / A \leq 0.8 \\
n=79\end{array}$ & $\begin{array}{c}\text { Group } 2 \\
0.8>E / A<2 \\
n=70\end{array}$ & $\begin{array}{c}\text { Group } 3 \\
E / A \geq 2 \\
n=13\end{array}$ & 1 vs 2 & 1 vs 3 & 2 vs 3 \\
\hline NHISS** & $\begin{array}{c}3.41 \pm 3.36 \\
{[0-16] \text { median } 3}\end{array}$ & $\begin{array}{c}5.43 \pm 5.92 \\
{[0-22] \text { median } 3}\end{array}$ & $\begin{array}{c}1.67 \pm 1.53 \\
{[0-3] \text { median } 2}\end{array}$ & 0.2388 & 0.3800 & 0.2874 \\
\hline Rankin** & $\begin{array}{c}2.16 \pm 1.67 \\
{[0-5] \text { median } 2}\end{array}$ & $\begin{array}{c}2.74 \pm 1.91 \\
{[0-6] \text { median } 3}\end{array}$ & $\begin{array}{c}2.33 \pm 2.08 \\
{[0-4] \text { median } 3}\end{array}$ & 0.0445 & 0.9419 & 0.6416 \\
\hline
\end{tabular}

** U Mann-Whitney Test; NIHSS - National Institute of Health Stroke Scale

the penumbra zone can be reduced in the presence of left ventricular dysfunction.

According to the results of the present study, an adverse effect on the neurological status on the 1st day after stroke was also brought about by impaired LV filling, indicating left ventricular diastolic dysfunction. The effect of LVDD was independent of coexisting LVLD and EF reduction. It has been previously shown that LVDD, independent of the coexistence of AF, increases the incidence of vascular events and deteriorates patients' status at 3 and 12 months after stroke [17]. The 
Table 4. Neurological status on the 1st day and functional status at 30 days after stroke, depending on $\mathrm{E} / \mathrm{A}$ values of contractile dysfunction and $\mathrm{EF}$

\begin{tabular}{|lccc|}
\hline & $\begin{array}{c}\text { Group B1 } \\
\mathbf{n}=\mathbf{2 2}\end{array}$ & $\begin{array}{c}\text { Group B2 } \\
\mathbf{n}=\mathbf{1 2 7}\end{array}$ & ;* \\
\hline Rankin & $3.32 \pm 1.81$ & $2.28 \pm 1.76$ & 0.0104 \\
NIHSS & $8.05 \pm 5.92$ & $3.68 \pm 4.31$ & 0.0004 \\
\hline
\end{tabular}

*U Mann-Whitney Test; NIHSS - National Institute of Health Stroke Scale

above observations concern patients with cryptogenic stroke. They can result from coexisting with LVDD undetected AF. LVDD is a recognised risk factor for AF, and it can be associated with the progression and symptom severity of AF, and it is also an indirect factor for systemic embolism [21, 22]. Together with LVDD, silent AF can also coexist. According to the results of the present study, E/A dysfunctions have a detrimental effect on patients' functional state one month after the onset of stroke. This sheds new light on the importance of diastolic dysfunction for the possibility of neurological state improvement following acute incidence of cerebral ischaemia. Many studies have shown that LVSD with low EF is only a risk factor for stroke, although there have been reports that do not support such a correlation [23-26]. According to the WARCEF trial, only $\mathrm{EF}<15 \%$ is associated with an increased risk of stroke (HR 2.125, 95\% CI, 1.182-3.818, p = 0.012) [27].

As we have demonstrated in this study, coexistence of low ejection fraction has been associated with adverse effect of LVDD on prognosis in the acute and subacute stage of cerebral infarct. Furthermore, the effect of LVDD varies depending on its severity.

In summary, the results of this study indicate that the presence of LVSD and/or LVDD is a worse prognostic factor in patients in the acute stage of stroke. It has been documented that heart dysfunction (HD) has an adverse effect on the functional status of patients in the chronic stage of stroke, and that this effect is independent of the treatment used, including thrombolysis [28-30].

The results obtained in this study also confirm the independent-of-therapy impact of HD. It is difficult to determine what percentage of stroke patients suffer from HD and are at risk of its decompensation, as well as in which patients HD is likely to have a significant impact on the neurological status. Over-activation of the vegetative system during stroke in patients with preexisting heart disorders may lead to exacerbation of cardiac dysfunction. Due to the differing definitions of $\mathrm{HD}$, prevalence data is inconsistent. Elderly patients with other comorbidities are in the higher risk group [29, 31].

The present study highlights the role of echocardiography in the structural and functional assessment of heart function and in finding abnormalities that may have clinical implications in stroke survivors. TTE may be useful in the planning of post-stroke rehabilitation due to the need to modify it in case of left ventricular dysfunction.

\section{Limitations}

The lack of an analysis of the impact of the type of stroke (aetiology) or the size of ischaemic focus on the stroke outcome.

\section{Conclusions}

Nearly one quarter of patients in the acute phase of stroke have features of systolic and / or diastolic dysfunction of the left ventricle.

Left ventricular dysfunction adversely affects both neurological status in the acute stage of stroke as well as functional status in the short-term follow-up.

Age at first stroke incidence and lower ejection fraction during the acute phase of illness are predictors of poor functional status one month after stroke.

\section{References}

1. murray C, Lopez A. Measuring the Global Burden of Disease. New England Journal of Medicine. 2013; 369(5): 448-457, doi: 10.1056/ nejmra1201534.

2. Sandhu RK, Hohnloser SH, Pfeffer MA, et al. Relationship between degree of left ventricular dysfunction, symptom status, and risk of embolic events in patients with atrial fibrillation and heart failure. Stroke. 2015; 46(3): 667-672, doi: 10.1161/STROKEAHA.114.007140, indexed in Pubmed: 25628308.

3. Granger C, Alexander J, McMurray J, et al. Apixaban versus Warfarin in Patients with Atrial Fibrillation. New England Journal of Medicine. 2011; 365(11): 981-992, doi: 10.1056/nejmoa1107039.

4. Liakos Cl, Grassos CA, Babalis DK, et al. European Society of Hypertension, European Society of Cardiology. 2013 ESH/ESC guidelines for the management of arterial hypertension: what has changed in daily clinical practice? High Blood Press Cardiovasc Prev. 2015; 22(1): 43-53, doi: 10.1007/s40292-014-0071-2, indexed in Pubmed: 25252743.

5. Gionfriddo MR, McCoy RG, Lipska KJ. The 2013 American Association of Clinical Endocrinologists' diabetes mellitus management recommendations: improvements needed. JAMA Intern Med. 2014; 174(2): 179-180, doi: 10.1001/jamainternmed.2013.12971, indexed in Pubmed: 24322834.

6. Catapano A, Graham I, Backer GDe, et al. 2016 ESC/EAS Guidelines for the Management of Dyslipidaemias. Eur Heart J. 2016; 37(39): 2999-3058, doi: 10.1093/eurheartj/ehw272.

7. Beneficial Effect of Carotid Endarterectomy in Symptomatic Patients with High-Grade Carotid Stenosis. New England Journal of Medicine. 1991; 325(7): 445-453, doi: 10.1056/nejm199108153250701.

8. Betti I, Castelli G, Barchielli A, et al. The role of N-terminal PRO-brain natriuretic peptide and echocardiography for screening asymptomatic left ventricular dysfunction in a population at high risk for heart failure. The PROBE-HF study. J Card Fail. 2009; 15(5): 377-384, doi: 10.1016/j.cardfail.2008.12.002, indexed in Pubmed: 19477397.

9. Mosterd A, Hoes AW, de Bruyne MC, et al. Prevalence of heart failure and left ventricular dysfunction in the general population; The Rotterdam Study. Eur Heart J. 1999; 20(6): 447-455, indexed in Pubmed: 10213348. 
10. McDonagh TA, Morrison CE, Lawrence A, et al. Symptomatic and asymptomatic left-ventricular systolic dysfunction in an urban population. Lancet. 1997; 350(9081): 829-833, doi: 10.1016/S0140-6736(97)03033-X, indexed in Pubmed: 9310600.

11. Kelly R, Struthers AD. Screening for left ventricular systolic dysfunction in patients with stroke, transient ischaemic attacks, and peripheral vascular disease. QJM. 1999; 92(6): 295-297, indexed in Pubmed: 10616704.

12. Asrar UI Haq M, Goh CY, Levinger I, et al. Clinical utility of exercise training in heart failure with reduced and preserved ejection fraction. Clin Med Insights Cardiol. 2015; 9: 1-9, doi: 10.4137/CMC.S21372, indexed in Pubmed: 25698883.

13. How to diagnose diastolic heart failure. European Heart Journal. 1998; 19(7): 990-1003, doi: 10.1053/euhj.1998.1057.

14. Echocardiographic Predictors of Stroke in Patients With Atrial Fibrillation. Archives of Internal Medicine. 1998; 158(12): 1316, doi: 10.1001/archinte.158.12.1316.

15. Hachet O, Guenancia C, Stamboul K, et al. Frequency and predictors of stroke after acute myocardial infarction: specific aspects of in-hospital and postdischarge events. Stroke. 2014; 45(12): 3514-3520, doi: 10.1161/STROKEAHA.114.006707, indexed in Pubmed: 25370585.

16. Kim D, Shim C, Hong GR, et al. Clinical Implications and Determinants of Left Atrial Mechanical Dysfunction in Patients With Stroke. Stroke. 2016; 47(6): 1444-1451, doi: 10.1161/strokeaha.115.011656.

17. Park HK, Kim BJ, Yoon CH, et al. Left Ventricular Diastolic Dysfunction in Ischemic Stroke: Functional and Vascular Outcomes. J Stroke. 2016; 18(2): 195-202, doi: 10.5853/jos.2015.01697, indexed in Pubmed: 27283279

18. Pullicino P, Mifsud V, Wong E, et al. Hypoperfusion-related cerebral ischemia and cardiac left ventricular systolic dysfunction. J Stroke Cerebrovasc Dis. 2001; 10(4): 178-182, doi: 10.1053/ jscd.2001.26870, indexed in Pubmed: 17903822.

19. Zannad F, Stough WG, Regnault V, et al. Is thrombosis a contributor to heart failure pathophysiology? Possible mechanisms, therapeutic opportunities, and clinical investigation challenges. Int J Cardiol. 2013; 167(5): 1772-1782, doi: 10.1016/j.jijcard.2012.12.018, indexed in Pubmed: 23298559.

20. Shantsila E, Wrigley BJ, Blann AD, et al. A contemporary view on endothelial function in heart failure. Eur J Heart Fail. 2012; 14(8): 873881, doi: 10.1093/eurjhf/hfs066, indexed in Pubmed: 22677484.

21. Yilmaz R, Demirbag R, Durmus I, et al. Association of stage of left ventricular diastolic dysfunction with $\mathrm{P}$ wave dispersion and oc- currence of atrial fibrillation after first acute anterior myocardial infarction. Ann Noninvasive Electrocardiol. 2004; 9(4): 330-338, doi: 10.1111/j.1542-474X.2004.94568.x, indexed in Pubmed: 15485510 .

22. Seo JY, Lee KB, Lee JG, et al. Implication of left ventricular diastolic dysfunction in cryptogenic ischemic stroke. Stroke. 2014; 45(9): 2757-2761, doi: 10.1161/STROKEAHA.114.006108, indexed in Pubmed: 25074516.

23. Gottdiener JS, McClelland RL, Marshall R, et al. Outcome of congestive heart failure in elderly persons: influence of left ventricular systolic function. The Cardiovascular Health Study. Ann Intern Med. 2002; 137(8): 631-639, indexed in Pubmed: 12379062.

24. Hays AG, Sacco RL, Rundek T, et al. Left ventricular systolic dysfunction and the risk of ischemic stroke in a multiethnic population. Stroke. 2006; 37(7): 1715-1719, doi: 10.1161/01. STR.0000227121.34717.40, indexed in Pubmed: 16741172.

25. Witt BJ, Brown RD, Jacobsen SJ, et al. Ischemic stroke after heart failure: a community-based study. Am Heart J. 2006; 152(1): 102-109, doi: 10.1016/j.ahj.2005.10.018, indexed in Pubmed: 16824838.

26. Freudenberger R, Hellkamp A, Halperin J, et al. Risk of Thromboembolism in Heart Failure. Circulation. 2007; 115(20): 2637-2641, doi: 10.1161/circulationaha.106.661397.

27. Di Tullio MR, Qian M, Thompson JLP, et al. WARCEF Investigators. Left Ventricular Ejection Fraction and Risk of Stroke and Cardiac Events in Heart Failure: Data From the Warfarin Versus Aspirin in Reduced Ejection Fraction Trial. Stroke. 2016; 47(8): 2031-2037, doi: 10.1161/ STROKEAHA.116.013679, indexed in Pubmed: 27354224.

28. Appelros P, Nydevik I, Viitanen M. Poor outcome after first-ever stroke: predictors for death, dependency, and recurrent stroke within the first year. Stroke. 2003; 34(1): 122-126, indexed in Pubmed: 12511762.

29. Ois A, Gomis M, Cuadrado-Godia E, et al. Heart failure in acute ischemic stroke. Journal of Neurology. 2008; 255(3): 385-389, doi: 10.1007/s00415-008-0677-1.

30. Abdul-Rahim AH, Fulton RL, Frank B, et al. VISTA collaborators. Associations of chronic heart failure with outcome in acute ischaemic stroke patients who received systemic thrombolysis: analysis from VISTA. Eur J Neurol. 2015; 22(1): 163-169, doi: 10.1111/ene.12548, indexed in Pubmed: 25370204.

31. Burkot J, Kopec G, Pera J, et al. Decompensated Heart Failure Is a Strong Independent Predictor of Functional Outcome After Ischemic Stroke. J Card Fail. 2015; 21(8): 642-646, doi: 10.1016/j.cardfail.2015.03.008, indexed in Pubmed: 25800549. 